\title{
Non-Gaussian Density Fluctuations from Entropically Generated Curvature Perturbations in Ekpyrotic Models
}

\author{
Jean-Luc Lehners ${ }^{1}$ and Paul J. Steinhardt ${ }^{1,2}$ \\ ${ }^{1}$ Princeton Center for Theoretical Physics, Princeton University, Princeton, NJ 08544 USA \\ ${ }^{2}$ Joseph Henry Laboratories, Princeton University, Princeton, NJ 08544, USA
}

\begin{abstract}
We analyze the non-gaussian density perturbations generated in ekpyrotic/cyclic models based on heterotic M-theory. In this picture, two scalar fields produce nearly scale-invariant entropic perturbations during an ekpyrotic phase that are converted into curvature modes after the ekpyrotic phase is complete and just before the big bang. Both intrinsic non-linearity in the entropy perturbation and the conversion process contribute to non-gaussianity. The range of the non-gaussianity parameter $f_{N L}$ depends on the details of the scalar field potential during the ekpyrotic phase, and on how gradual the conversion process is. Although a wider range is possible, in principle, natural values of the parameters of the potential combined with a gradual conversion process lead to values of $-60 \lesssim f_{N L} \lesssim+80$, typically much greater than slow-roll inflation but within the current observational bounds.
\end{abstract}

Ekpyrotic [1] and cyclic [2] models of the universe use quantum fluctuations of scalar fields produced during a slowly contracting phase with equation of state $w>1$ to generate the observed nearly scale-invariant spectrum of curvature (energy density) fluctuations after the big bang. One mechanism considered for converting the fluctuations of a scalar field into cosmological curvature perturbations relies on higher-dimensional effects at the collision between orbifold planes (branes) along an extra dimension [3]. Recently, however, a new "entropic" mechanism $[4,5]$ has been proposed that relies on two (or more) scalar fields and ordinary $4 \mathrm{~d}$ physics, stimulating new approaches to ekpyrotic and cyclic cosmology that may not require branes or extra dimensions at all [6-9].

In this paper, we wish to consider an important byproduct of the entropic mechanism: a non-gaussian contribution to the density fluctuation spectrum that is more than an order of magnitude greater than for conventional inflationary models and that can satisfy current observational bounds, including the recently claimed detection of non-gaussianity [10]. Our results differ significantly from the cases considered by Koyama et al.[11] and Buchbinder et al.[12] in which they assumed an ekpyrotic $(w \gg 1)$ phase that continues all the way to the conversion of entropic to curvature fluctuations, as in the "new ekpyrotic" model [6]; for these cases, the non-gaussianity is amplified by the non-linear evolution on super-horizon scales to the point where $f_{N L}$, the parameter characterizing the non-linear curvature perturbation [13], reaches magnitude $\mathcal{O}\left(c_{1}^{2}\right)$, where $c_{1} \approx 2 \sqrt{w+1}$ parameterizes the steepness of the scalar field potential during the ekpyrotic phase. A potential problem is that a minimum of $c_{1} \geq 10$ is required just to satisfy the current upper bound constraints on $n_{s}$, the spectral tilt of the scalar (energy density) perturbation spectrum [5]; and $c_{1} \geq 30$ is needed to reach the best-fit value $n_{s} \approx 0.97$. Yet, excluding finelytuned cancellations, the resulting value of $f_{N L}$ obtained in Ref. [6-9] is marginally consistent with current observational bounds on non-gaussianity only for $c_{1}$ restricted to a narrow range $(\lesssim 15)$.

Here we show that, by having the conversion occur af- ter the ekpyrotic phase, as is natural from the point of view of heterotic M-theory and the cyclic model $[2,5]$, the non-gaussianity is reduced and much less sensitive to $c_{1}$, so that one can naturally fit the best-fit value of $n_{s}$ and current bounds on both $f_{N L}$ at the same time. More specifically, the net value of $f_{N L}$ depends on both the intrinsic non-linearity in the entropy perturbation as it is generated during the ekpyrotic phase as well as the duration of the conversion process. These two contributions to $f_{N L}$ compete with each other, in that the intrinsic nonlinearity, which depends on the steepness of the scalar field potential, is always positive and the contribution depending on the duration of the conversion process is negative. In principle, with arbitrarily steep potentials and arbitrarily sharp conversions, a wide range of $f_{N L}$ is possible, and one can even have a finely-tuned cancellations of two large but oppositely signed contributions that give nearly zero non-gaussianity. Excluding these fine-tuned cases, though, we find that $f_{N L}$ lies within the current observationally favored range of $26.9<f_{N L}<146.7$ that was recently reported at $2 \sigma[10]$ or the bound obtained earlier by the WMAP collaboration, $-36<f_{N L}<100$ [14], while, at the same time, matching the best-fit value of $n_{s}$ allowed by WMAP.

As a concrete example, we will analyze the $4 \mathrm{~d}$ effective field theory derived from the ekpyrotic/cyclic model of [5]. The $4 \mathrm{~d}$ theory describes the phase when two boundary branes approach one another and collide [15] in heterotic M-theory [16]; for a short review see [17]. The brane worldvolume is described by gravity and two canonically normalized scalar fields, $\phi_{1,2}$, which parameterize the distance between the two branes as well as the volume of the internal Calabi-Yau space [15]. For the purposes of this paper, though, the connection to M theory is not essential; it simply provides physical motivation for considering this particular $4 \mathrm{~d}$ field theoretic example. The same effects will occur in other $4 \mathrm{~d}$ field theories in which the conversion of entropic to curvature perturbations occurs after the ekpyrotic $(w \gg 1)$ phase.

We assume a flat Friedmann-Robertson-Walker (FRW) background with line element $\delta s^{2}=-\delta t^{2}+a^{2}(t) \delta \mathbf{x}^{2}$ and 


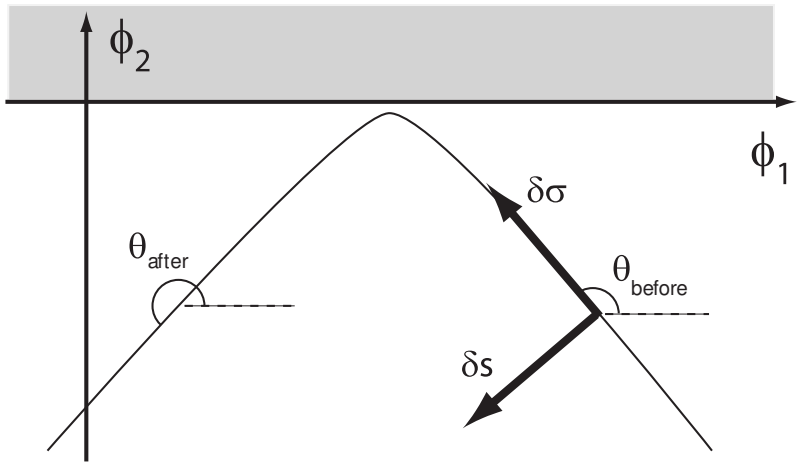

FIG. 1: The background trajectory in scalar field space is straight everywhere except for a brief period after the ekpyrotic phase around the time of reflection. The shaded region indicates the geometrically forbidden region bounded by the axis $\phi_{2}=0$. In the presence of brane-bound matter, the trajectory reflects smoothly off this boundary. Also shown are the directions of adiabatic $(\delta \sigma)$ and entropic $(\delta s)$ fluctuations, as well as the angle of the trajectory before and after the reflection.

scale factor $a(t)$, and steep, negative, scalar field potentials of the form

$$
V\left(\phi_{1}, \phi_{2}\right)=-V_{1} e^{-\int c_{1} \delta \phi_{1}}-V_{2} e^{-\int c_{2} \delta \phi_{2}},
$$

where $c_{1}=c_{1}\left(\phi_{1}\right), c_{2}=c_{2}\left(\phi_{2}\right)$ and $V_{1}, V_{2}$ are positive constants. During the ekpyrotic phase, the $c_{i}$ are nearly constant and $c_{i} \gg 1$. Then the Einstein-scalar equations admit a scaling solution

$$
a=(-t)^{1 / \epsilon}, \quad \phi_{i}=\frac{2}{c_{i}} \ln \left(-\sqrt{c_{i}^{2} V_{i} / 2} t\right), \quad \frac{1}{\epsilon}=\sum_{i} \frac{2}{c_{i}^{2}},
$$

which describes a very slowly contracting universe with $\epsilon \gg 1$ and $w+1=2 \epsilon / 3 \gg 1$ - the defining characteristics of an ekpyrotic phase. At the end of the ekpyrotic phase, the effective values of $c_{i} \rightarrow 0$ and the ekpyrotic potential energy ceases to be important cosmologically.

The colliding branes solution corresponds to an almost everywhere straight line trajectory in scalar field space in the $4 \mathrm{~d}$ effective theory. The only deviation from this straight trajectory is one required by the fact that the scalar field moduli space admits a boundary at $\phi_{2}=0$, which from the higher-dimensional point of view corresponds to the locus where both the scale factor on the negative-tension brane and the volume of the Calabi-Yau manifold reach zero. As shown in [15], the trajectory simply gets reflected at this boundary. In the presence of matter on the branes, the reflection is smoothed out by an effective repulsive potential [15] and the trajectory never actually touches the boundary, as shown in Fig. 1. This smooth reflection is relevant for the density fluctuation spectrum, because it suffices to induce the conversion from entropic to curvature perturbations without introducing any extra elements to the theory. In fact, one could even imagine that it is the effective potential associated with this reflection which causes the ekpyrotic phase to end. In any case, the conversion is followed by a period of scalar field kinetic energy domination, which sets simple cosmological conditions near the big crunch/big bang transition [2].

It is useful to recast the evolution in terms of the adiabatic variable $\sigma$ (pointing along the background trajectory) and the entropy variable $s$, pointing transverse to the trajectory (see Fig. 1) [18]. Up to unimportant additive constants that will be fixed shortly, we define

$$
\begin{aligned}
\sigma & \equiv \frac{\dot{\phi}_{1} \phi_{1}+\dot{\phi}_{2} \phi_{2}}{\dot{\sigma}} \\
s & \equiv \frac{\dot{\phi}_{1} \phi_{2}-\dot{\phi}_{2} \phi_{1}}{\dot{\sigma}},
\end{aligned}
$$

where $\dot{\sigma} \equiv \sqrt{\dot{\phi}_{1}^{2}+\dot{\phi}_{2}^{2}}(\dot{\sigma}$ is positive by definition). A dot represents a derivative with respect to FRW time. Then we can expand the potential up to third order as follows [20]:

$$
V_{e k}=-V_{0} e^{\sqrt{2 \epsilon} \sigma}\left[1+\epsilon s^{2}+\frac{\kappa_{3}}{3 !} \epsilon^{3 / 2} s^{3}\right],
$$

where $\kappa_{3}$ is of $\mathcal{O}(1)$ for typical potentials (the case of exact exponentials corresponds to $\left.\kappa_{3}=-4 \sqrt{2 / 3}\right)$. The scaling solution (2) can be rewritten as

$$
a(t)=(-t)^{1 / \epsilon} \quad \sigma=-\sqrt{\frac{2}{\epsilon}} \ln \left(-\sqrt{\epsilon V_{0}} t\right) \quad s=0 .
$$

The perturbations about this contracting universe have a growing mode - the entropy perturbation - corresponding to the relative fluctuation of the two fields: $\delta s \equiv\left(\dot{\phi}_{1} \delta \phi_{2}-\dot{\phi}_{2} \delta \phi_{1}\right) / \dot{\sigma}$, We will decompose the entropy perturbation into a linear, gaussian part and a secondorder perturbation by writing $\delta s=\delta s^{(1)}+\delta s^{(2)}$. Its equation of motion, on large scales and up to second order in field perturbations is then given by [18]

$$
\begin{aligned}
& \ddot{\delta s}+3 H \dot{\delta s}+\left(V_{s s}+3 \dot{\theta}^{2}\right) \delta s \\
& +\frac{\dot{\theta}}{\dot{\sigma}}\left(\dot{\delta s}^{(1)}\right)^{2}+\frac{2}{\dot{\sigma}}\left(\ddot{\theta}+\dot{\theta} \frac{V_{\sigma}}{\dot{\sigma}}-\frac{3}{2} H \dot{\theta}\right) \delta s^{(1)} \dot{\delta s}^{(1)} \\
& +\left(\frac{1}{2} V_{s s s}-\frac{5 \dot{\theta}}{\dot{\sigma}} V_{s s}-\frac{9 \dot{\theta}^{3}}{\dot{\sigma}}\right)\left(\delta s^{(1)}\right)^{2}+\frac{2 \dot{\theta}}{\dot{\sigma}} \delta \epsilon^{(2)}=0(.7)
\end{aligned}
$$

Here $H \equiv \dot{a} / a$ is the Hubble parameter, $V_{\sigma}$ denotes a derivative of the potential along the background trajectory and $V_{s \ldots s}$ denote successive derivatives along the $\delta s$ direction. The angle $\theta$ of the background trajectory is defined as in [19] by $\cos (\theta)=\dot{\phi}_{1} / \dot{\sigma}, \sin (\theta)=\dot{\phi}_{2} / \dot{\sigma}$. A useful expression for the time variation of this angle is $\dot{\theta}=-V_{s} / \dot{\sigma}$. In the simplest models, the background scalar field trajectory is well-approximated as a straight line [15], $\dot{\theta}=0$. In this case, we have $\dot{\phi}_{2}=\gamma \dot{\phi}_{1}$, which implies $c_{1}=\gamma c_{2}$. For the colliding branes solution with 
empty branes, $\gamma=\frac{1}{\sqrt{3}} \theta_{H}\left(t-t_{r e f}\right)$, where $\theta_{H}$ is the Heaviside step function (with values \pm 1 for $t \gtrless t_{r e f}$ ) and $t_{r e f}$ denotes the time of the reflection, see [15] for details. The last term in equation (7) is a non-local term proportional to the difference in spatial gradients between the linear entropy perturbation and its time derivative. This difference evolves as $a^{-3}$ [18], so that it remains approximately constant during the ekpyrotic phase when $a$ is very slowly varying. This ends up being exponentially suppressed compared to the entropy perturbation itself, which grows by a factor of $10^{30}$ or more during this same period [5]. After the ekpyrotic phase has ended, the non-local term grows because $a \propto(-t)^{-1 / 3}$, but this growth is negligible compared to the exponential suppression during the ekpyrotic phase. Hence, the non-local term can be safely neglected. For analyzing the equation of motion, it is convenient to use conformal time $\tau$. Denoting $\tau$ derivatives with primes, and introducing the re-scaled entropy field $\delta S=a(\tau) \delta s$, to first order in perturbations, equation (7) reduces to $\delta S_{L}^{\prime \prime}+\left(k^{2}-\frac{a^{\prime \prime}}{a}+a^{2} V_{s s}\right) \delta S_{L}=0$. The fastroll parameter $\epsilon \equiv \dot{\sigma}^{2} /\left(2 H^{2}\right) \approx c_{1}^{2} /\left(2\left(1+\gamma^{2}\right)\right)$ defines the equation of state of the background scaling solution in the ekpyrotic contraction phase. In terms of $\epsilon$ and its derivative with respect to the number $\mathcal{N}=\ln \left(a_{\text {end }} H_{\text {end }} / a H\right)$ of e-folds remaining before the end of the ekpyrotic phase, the solution to the equation of motion at long wavelengths and linear order is

$$
\delta S_{L}=f_{L}(k, \epsilon)(-\tau)^{-1+1 / \epsilon-\frac{d \ln \epsilon}{2 d \mathcal{N}}} .
$$

Since $\epsilon \propto(w+1) \gg 1$ during the ekpyrotic contraction phase, this leads to a nearly scale-invariant spectrum with index [5] $n_{s}=1+\frac{2}{\epsilon}-\frac{d \ln \epsilon}{d \mathcal{N}}$. As discussed in [5], achieving a red spectral tilt consistent with best-fit measurement $\left(n_{s} \approx 0.97\right)$ typically requires $\epsilon \sim \mathcal{N}^{1.5-2}$ or $c_{1} \gtrsim 30$.

On large scales and at linear order, the comoving curvature perturbation evolves according to [19] $\dot{\mathcal{R}}=\frac{2 H}{\dot{\sigma}} \dot{\theta} \delta s$. Thus, the entropy perturbation generates a curvature perturbation when the background trajectory bends at the reflection. Moreover, this curvature perturbation is of the right amplitude if the potentials turn off at a time $t_{\text {end }} \approx-10^{3} M_{P l}^{-1}$, where $M_{P l}$ is the Planck mass [5].

Next we consider the non-linearities responsible for the non-gaussianity of the perturbation spectrum. The intrinsic non-gaussianity in the entropy perturbation is produced during the ekpyrotic phase and can be determined from the equation of motion for the entropy field to leading order

$$
\delta S^{\prime \prime}+\left(k^{2}-\frac{2}{\tau^{2}}\right) \delta S+\frac{1}{2} a V_{s s s}(\delta S)^{2}=0 .
$$

The last term is approximately

$$
\frac{1}{2} a V_{s s s} \approx-\frac{\kappa_{3}}{2(-\tau)^{2}} \sqrt{\epsilon}
$$

The solution to the equation of motion (9), at long wavelengths and up to second order in field perturbations, is then given by

$$
\begin{aligned}
\delta S & =\delta S_{L}+\tilde{c}\left(\delta S_{L}\right)^{2}, \\
\tilde{c} & =\frac{\kappa_{3} \sqrt{\epsilon}}{8} .
\end{aligned}
$$

So $\tilde{c}$ characterizes the intrinsic non-linearity in the entropy perturbation. As a reference, we note that for empty branes, and with the original potential (1), i.e. with constant $c_{1}=-c_{2} / \sqrt{3}$, we have $\kappa_{3}=-4 \sqrt{2 / 3}$ hence $\tilde{c}=-\sqrt{\epsilon / 6}=-c_{1} / 4$.

The time evolution of the curvature perturbation to second order in field perturbations and at long wavelengths is given in FRW time by [18]:

$$
\dot{\mathcal{R}}=\frac{2 H}{\dot{\sigma}} \dot{\theta} \delta s+\frac{H}{\dot{\sigma}^{2}}\left[-\left(V_{s s}+4 \dot{\theta}^{2}\right)\left(\delta s^{(1)}\right)^{2}+\frac{V_{, \sigma}}{\dot{\sigma}} \delta s \dot{\delta} s\right] .
$$

At linear order a bending $(\dot{\theta} \neq 0)$ of the background trajectory combined with a non-zero entropy perturbation source the curvature perturbation on large scales and result in a linear, gaussian curvature perturbation

$$
\mathcal{R}_{L}=\int \frac{2 H}{\dot{\sigma}} \dot{\theta} \delta s^{(1)} .
$$

During this process of conversion, the entropy perturbation evolves according to Eq. (7) in a rather complicated way, which is why the above integral and the following calculation have to be performed numerically. Qualitatively, a sharp reflection leads to a drastic diminution in the entropy perturbation and a much smaller value of the above integral, while a smooth and more gradual reflection results in an enhanced efficiency of conversion.

The leading non-gaussianity is generated when modes are outside the horizon, so the non-gaussianity is of the local, wavelength-independent type [13, 21, 22]. The nonlinearity parameter $f_{N L}$ is then defined by the relations [13] $\Phi_{H}=\Phi_{L}+f_{N L} \Phi_{L}^{2}$, where $\Phi_{H}$ is Bardeen's spacespace metric perturbation [23] and $\Phi_{L}$ is its value to linear order. We adopt the convention of Ref. [18] where the comoving curvature perturbation is equal but opposite in sign to Bardeen's $\zeta$ variable on large scales, so that during matter domination we have $\mathcal{R}=-\frac{5}{3} \Phi_{H}$, and thus $\mathcal{R}=\mathcal{R}_{L}-\frac{3}{5} f_{N L} \mathcal{R}_{L}^{2}$. (Note that this definition of $f_{N L}$ agrees with Komatsu and Spergel [13] and WMAP [14] but is opposite in sign to Maldacena [22].)

At the quadratic level, Eq. (13) then implies that there are three distinct ways in which the curvature perturbation can acquire non-linear contributions:

(1) Before the reflection, the terms proportional to $V_{s s}$ and $V_{, \sigma}$ create non-linearities in $\mathcal{R}$ during the entire ekpyrotic phase in which the entropy perturbation is generated; during this phase, the background trajectory is a straight line, so Eq. (13) reduces to $\dot{\mathcal{R}}=\frac{H}{\dot{\sigma}^{2}}\left(-V_{s s}(\delta s)^{2}+\right.$ $\left.\frac{V, \sigma}{\dot{\sigma}} \delta s \dot{\delta} s\right)$, which to leading order reads $\dot{\mathcal{R}}=\frac{1}{2} f_{L}^{2}(-t)^{-3}$. The integrated contribution thus amounts to

$$
\mathcal{R}_{\text {integrated }}=-\frac{1}{4}\left(\delta s_{\text {end }}^{(1)}\right)^{2} .
$$


This term leads to

$$
f_{N L}^{\text {integrated }}=+\frac{5}{12 \mathcal{R}_{L}^{2}}\left(\delta s_{\text {end }}^{(1)}\right)^{2},
$$

and is typically small, of order $O(10)$ or less.

(2) At the reflection, any intrinsic non-linearity in $\delta s$ will be converted into a non-linearity in $\mathcal{R}$ due to the first term in (13); thus we get

$$
f_{N L}^{\text {intrinsic }}=-\frac{5}{3 \mathcal{R}_{L}^{2}} \int \frac{2 H}{\dot{\sigma}} \dot{\theta} \delta s^{(2)} .
$$

This term is positive and increases with increasing $\tilde{c}$, although generally not quite as fast as linearly because of the non-linear evolution of $\delta s^{(2)}$.

(3) Also at the reflection, the terms involving $\delta s^{(1)}$ lead to non-linearities in $\mathcal{R}$ according to

$$
\begin{aligned}
& f_{N L}^{\text {reflection }}=\frac{5}{3 \mathcal{R}_{L}^{2}} \int_{\text {ref }} \frac{H}{\dot{\sigma}^{2}} \quad\left[\left(V_{s s}+4 \dot{\theta}^{2}\right)\left(\delta s^{(1)}\right)^{2}\right. \\
& \left.-\frac{V_{, \sigma}}{\dot{\sigma}} \delta s \dot{\delta} s\right] .
\end{aligned}
$$

In order to close the equations for the purposes of this study, we are modeling the reflection in terms of an effective potential $V$ that acts when $\phi_{2}$ gets small and causes it to reflect; we find that the results do not depend sensitively on the form of $V$ except for how gradual the reflection is. Even disregarding the $\mathcal{R}_{L}^{-2}$ prefactor, this term generically increases in magnitude as the reflection is made sharper and it is always negative in sign.

The total $f_{N L}$ is the sum of all the above contributions. In Fig. 2, we have shown the results for a typical value $\epsilon=36$ (corresponding to e.g. $c_{1}=c_{2}=12$ ) and for the range $-5 \leqslant \kappa_{3} \leqslant 5$ with the lower curves corresponding to $\kappa_{3}=-5$ and the higher curves to $\kappa_{3}=+5$. In order to see how robust the results are, we experiment with different potential forms to model the reflection of $\phi_{2}$. The results shown here are for the potential forms $V_{1}\left(\phi_{2}\right)=$ $v\left(\phi_{2}^{-2}+r \phi_{2}^{-6}\right)$ and $V_{2}\left(\phi_{2}\right)=v\left[\left(\sinh \phi_{2}\right)^{-2}+r\left(\sinh \phi_{2}\right)^{-4}\right]$, where we have varied the coefficient $v$ over several orders of magnitude and taken $r=0,1$ in each case. Each potential is represented in Fig. 2 by a different curve, with the $r=0$ examples corresponding to the curves bounding the shaded area. We will not give further details here because, as we will show, they are not important.

We find that the results for $f_{N L}$ fall into two distinct regimes depending mainly on the duration of the conversion process. We measure the duration by specifying the number of e-folds ('Hubble times') by which the scale factor $a$ shrinks during the process of conversion; it is useful to distinguish the case of "rapid conversion," where the conversion lasts at most about 0.2 Hubble times, from the case of "gradual conversion," where the conversion lasts on the order of 1 Hubble time (The details of the reflection affect the direction of the trajectory afterwards, which has to be taken into account when incorporating this conversion mechanism within the full cosmological model; but this is beyond the scope of this paper.)

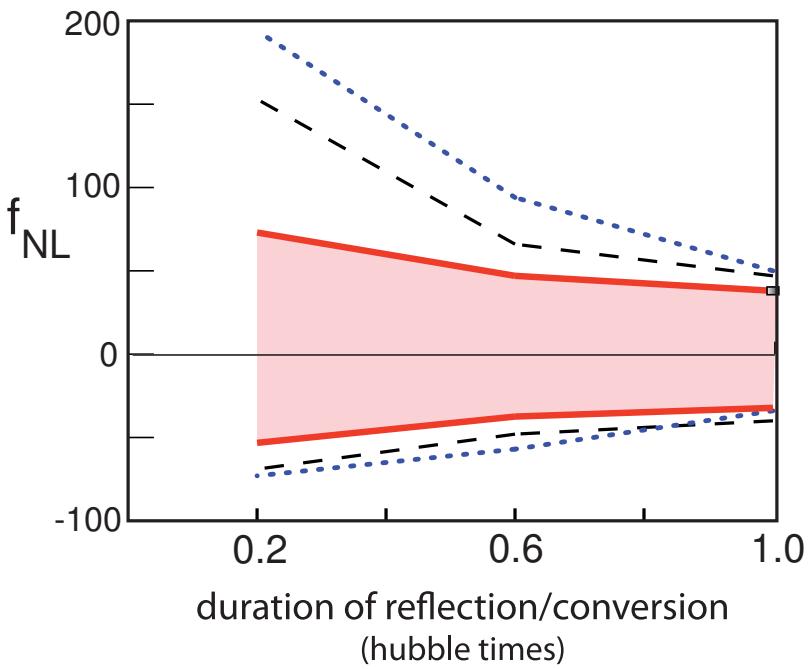

FIG. 2: The predicted value of the non-gaussianity parameter $f_{N L}$ as a function of the duration of the reflection. The shaded range represents results for the simplest ekpyrotic and reflection potentials allowing $\kappa_{3}$ to range from +5 (top) to -5 (bottom). The upper and lower boundaries can be extended (dashed and dotted curves) by carefully tuning the reflection potentials, but the change is not significant for typical durations $\mathcal{O}(1)$.

In the case of rapid conversion, the conversion is inefficient so that the leading order curvature contribution $\mathcal{R}_{L}$ is smaller by a factor of $\mathcal{O}(10)$ or more compared to the case of gradual conversions. Reducing $\mathcal{R}_{L}$ by a factor of ten automatically amplifies all the contributions to $f_{N L}$ by a factor of $\mathcal{O}(100)$, although the summing up of all the contributing terms cancels out some of the amplification. The net result for $f_{N L}$ turns out to be of $\mathcal{O}(100)$ or more, as shown in Fig. 2 for the case when the duration of the conversion is only 0.2 Hubble times. The figure also shows that the result is sensitive to the form of the potential and the potential parameters $(v$ and $r$ ) used to model the reflection. However, much of the entire range lies outside the observationally acceptable range, and so can be dropped from further consideration. Here we are chiefly interested in making general predictions, and not in the accidental cancellation of large numbers, which would clearly be fine-tuned and unattractive.

The second regime is where the reflection and conversion process are gradual. In these cases, the conversion is efficient and all contributions to $f_{N L}$ tend to be reduced. Roughly, for $\kappa_{3}$ ranging from -5 to 5 and for typical values of $\epsilon \sim 50$, we find that the predictions converge to a range

$$
-60 \lesssim f_{N L} \lesssim+80
$$

with very weak dependence on the parameters of the reflection potential. If $\epsilon$ is pushed to higher values, e.g. up to $\epsilon=200$, this range expands to $-120 \lesssim f_{N L} \lesssim+160$. Analytic estimates of the above calculations confirm that 
the results are rather insensitive to the specific form of the potential causing the reflection, but depend most strongly on the duration of conversion as well as roughly linearly on $\kappa_{3} \sqrt{\epsilon}$ [24].

Several comments are in order. First, our results show that, in the ekpyrotic model, it is very unnatural to get a value of $\left|f_{N L}\right| \lesssim 1$, the value obtained for slow-roll inflation [22]. The contributions to the local $f_{N L}$ are generally greater than ten so that small values can only be achieved by accidental cancellation of large terms.

Conversely, obtaining large local $f_{N L}$ is completely non-generic for inflation, requiring the finely-tuned addition of extra fields and interactions. When the fields and interactions are introduced, there is no predictive convergence to a bounded range for $f_{N L}$. The result can be anything from negligible $f_{N L}$ to values of either sign and extending orders of magnitude beyond current bounds. By contrast, in the ekpyrotic model, conversion of scalar fluctuations to curvature perturbations is absolutely required, and, at least for one proven conversion mechanism, generically generates a measurable
$f_{N L}$. We have found that a rapid conversion, whether in an ekpyrotic or kinetic energy dominated phase, generically leads to a large $\left|f_{N L}\right|=\mathcal{O}(100)$ or greater that is already ruled out observationally. Furthermore, if the conversion occurs in the ekyprotic phase, it is difficult to obtain simultaneously an observationally acceptable $f_{N L}$ and an observationally acceptable spectral tilt. On the other hand, a wide range of ekpyrotic/cyclic models satisfying current constraints on the spectral tilt generate a measurable non-gaussianity consistent with current observations if the conversion is gradual and occurs after the ekpyrotic phase is completed.

Acknowledgements We would like to thank T. Battefeld, D. Baumann, J. Khoury, E. Komatsu, N. Turok, F. Vernizzi for useful discussions and particularly K. Koyama and D. Wands for remarks that pointed us to the differences between rapid and gradual conversion, and S. Renaux-Petel for pointing out a sign error in an earlier version of this paper. This work is supported in part by the US Department of Energy grant DE-FG0291ER40671.
[1] J. Khoury, B. A. Ovrut, P. J. Steinhardt, and N. Turok, Phys. Rev. D64, 123522 (2001).

[2] P. J. Steinhardt and N. Turok, Science 296, 1436 (2002); Phys. Rev. D65, 126003 (2002).

[3] A. J. Tolley, N. Turok and P. J. Steinhardt, Phys. Rev. D 69, 106005 (2004); P. L. McFadden, N. Turok and P. J. Steinhardt, Phys. Rev. D 76, 104038 (2007).

[4] A. Notari and A. Riotto, Nucl. Phys. B 644, 371 (2002); F. Di Marco, F. Finelli and R. Brandenberger, Phys. Rev. D 67, 063512 (2003).

[5] J. L. Lehners, P. McFadden, N. Turok and P. J. Steinhardt, Phys. Rev. D 76, 103501 (2007).

[6] E. I. Buchbinder, J. Khoury and B. A. Ovrut, Phys. Rev. D 76, 123503 (2007).

[7] P. Creminelli and L. Senatore, JCAP 0711, 010 (2007).

[8] K. Koyama and D. Wands, JCAP 0704, 008 (2007); K. Koyama, S. Mizuno and D. Wands, Class. Quant. Grav. 24, 3919 (2007).

[9] T. J. Battefeld (2007), arXiv:0710.2540 [hep-th].

[10] A. P. S. Yadav and B. D. Wandelt, arXiv:0712.1148 [astro-ph].

[11] K. Koyama, S. Mizuno, F. Vernizzi and D. Wands, JCAP 0711, 024 (2007).

[12] E. I. Buchbinder, J. Khoury, and B. A. Ovrut (2007),
arXiv:0710.5172 [hep-th].

[13] E. Komatsu and D. N. Spergel, arXiv:astro-ph/0012197; Phys. Rev. D 63, 063002 (2001).

[14] D. N. Spergel et al. (2006), astro-ph/0603449.

[15] J.-L. Lehners, P. McFadden, and N. Turok, Phys. Rev. D75, 103510 (2007); J.-L. Lehners, P. McFadden, and N. Turok, Phys. Rev. D76, 023501 (2007); J.-L. Lehners and N. Turok, Phys. Rev. D77, 023516 (2008).

[16] A. Lukas, B. A. Ovrut, K. S. Stelle, and D. Waldram, Phys. Rev. D59, 086001 (1999).

[17] J. L. Lehners, AIP Conf. Proc. 957, 337 (2007), arXiv:0708.2633 [hep-th].

[18] D. Langlois and F. Vernizzi, JCAP 0702, 017 (2007).

[19] C. Gordon, D. Wands, B. A. Bassett, and R. Maartens, Phys. Rev. D63, 023506 (2001).

[20] E. I. Buchbinder, J. Khoury and B. A. Ovrut, JHEP 0711, 076 (2007) [arXiv:0706.3903 [hep-th]].

[21] E. Sefusatti and E. Komatsu, Phys. Rev. D 76, 083004 (2007).

[22] J. M. Maldacena, JHEP 0305, 013 (2003).

[23] J. M. Bardeen, Phys. Rev. D 22, 1882 (1980).

[24] J.-L. Lehners and P. J. Steinhardt, in preparation. 